\title{
Colorimetric detection of morphine in a molecularly imprinted polymer using an aqueous mixture of $\mathrm{Fe}^{3+}$ and $\left[\mathrm{Fe}(\mathrm{CN})_{6}\right]^{3-\text { it }}$
}

\author{
Huan-Cheng Hsu, Lin-Chi Chen, Kuo-Chuan Ho* \\ Department of Chemical Engineering, National Taiwan University, Taipei 10617, Taiwan
}

Received 6 January 2003; received in revised form 22 October 2003; accepted 7 November 2003

\begin{abstract}
In this work, a molecularly imprinted polymer (MIP) of morphine (MO) was prepared through thermal radical copolymerization of methacrylic acid (MAA) and ethylene glycol dimethacrylate (EDMA) in the presence of MO templates, and a molecularly imprinted sorbent assay (MIA) based on a colorimetric reporter was developed to determine the adsorption isotherm of MO-MIP binding. In practice, the MO-bound MIP was brought into contact with an aqueous mixture of $\mathrm{Fe}^{3+}$ and $\left[\mathrm{Fe}(\mathrm{CN})_{6}\right]^{3-}$ so that the 3-phenolic group of MO was oxidized and $\mathrm{Fe}^{3+}$ was reduced to $\mathrm{Fe}^{2+}$. As a result, the MO-bound MIP was stained with Prussian blue (PB), which was attributed to the instant co-precipitation of $\mathrm{Fe}^{2+}$ and $\left[\mathrm{Fe}(\mathrm{CN})_{6}\right]^{3-}\left(K_{\mathrm{sp}}=10^{-40}\right)$. Accordingly, MO-MIP binding of the blue dye could be detected by visible spectroscopy. In addition, such staining could successfully distinguish MO from codeine. Upon data analyses, a two-site binding isotherm with two dissociation constants of $6.00 \times 10^{-5}$ and $1.03 \times 10^{-3} \mathrm{M}$ was found for MO-MIP binding. MIAs for non-MIP were also performed. In addition, the results of flow-system characterizations and the particle size effect are also described in this paper.

(c) 2003 Elsevier B.V. All rights reserved.
\end{abstract}

Keywords: Colorimetric detection; Molecularly imprinted polymer (MIP); Molecularly imprinted sorbent assay (MIA); Morphine (MO); Prussian blue (PB)

\section{Introduction}

Morphine (MO) is a frequently used drug for relieving severe pain of patients, but excessive or habitual uptake can cause toxic symptoms. To prevent overdose-induced toxication, the sensitive monitoring of MO concentrations in a patient's blood or urine is necessary. It was reported that around $90 \%$ of orally administrated MO is excreted in urine within $24 \mathrm{~h}$ and around $10 \%$ of the excreted MO remains un-metabolized. Typical MO concentrations in a patient's urine were thus evaluated to be less than $1 \mu \mathrm{g} / \mathrm{ml}(1 \mathrm{ppm})$ [1]. To date, many analytical techniques for MO detection, as summarized in Table 1, have been developed [2-11] to satisfy the requirements of sensitivity, specificity, and reliability. Although many detection modes can be used to quantify the amounts of MO, strategies to achieve its spe-

\footnotetext{
is Presented at the 2nd International Workshop on Molecularly Imprinted Polymers, Montpellier, 15-19 September 2002.

$*$ Corresponding author. Tel.: +886-2-2366-0739; fax: +886-2-2362-3040.

E-mail address: kcho@ntu.edu.tw (K.-C. Ho).
}

cific recognition are relatively few. Most techniques adopt high performance liquid chromatography (HPLC) [2-7], which can readily discriminate impurities and provide high sensitivities. Some approaches function based on enzymatic immunoassays $[8,9]$. Yet, the costs and stability of enzymes involved in the above techniques are shortcomings. Thus, the use of molecularly imprinted polymers (MIP) [10], which fulfill both economic and stability criteria, is considered a more reliable and robust strategy for specific binding.

Molecular imprinting mimics the molding process in which an imprinting molecule serves as a template for the criterion of a substrate-selective macromolecular matrix [12]. Hence, the template molecule and MIP are regarded as a biomimetic antigen and antibody, respectively. It has been found that the MIP of MO can be prepared by a non-covalent imprinting approach, in which radical copolymerization of methacrylic acid (MAA) (the functional monomer) and ethylene glycol dimethacrylate (EDMA; the cross-linking agent) is carried out in the presence of MO templates $[10,13,14]$. To demonstrate the specific recognition of the template molecule by MIP and/or to 
Table 1

A partial list of analytical techniques for the MO detection reported in literature

\begin{tabular}{lll}
\hline Detection method & Limit of detection & Reference \\
\hline HPLC $^{\text {a-UV-Vis spectroscopy }}$ & $5 \mathrm{ng} / \mathrm{ml}$ & {$[2]$} \\
HPLC-fluorescence & $300 \mathrm{ng} / \mathrm{ml}$ & {$[3]$} \\
HPLC-chemiluminescence & $50 \mathrm{ng} / \mathrm{ml}$ & {$[4]$} \\
HPLC-UV-ED $^{\mathrm{b}}$ & $50 \mathrm{ng} / \mathrm{ml}$ & {$[5]$} \\
HPLC-amperometry & $1 \mathrm{ng} / \mathrm{ml}$ & {$[6]$} \\
HPLC-coulometry & $0.1 \mathrm{ng} / \mathrm{ml}$ & {$[7]$} \\
Radio immunoassay (RIA) & $3 \mathrm{ng} / \mathrm{g} \mathrm{blood}$ & {$[8]$} \\
Immunoassay with SPR & $0.1 \mathrm{ng} / \mathrm{ml}$ & {$[9]$} \\
MIPd-competitive amperometry & $50 \mathrm{ng} / \mathrm{ml}$ & {$[10]$} \\
Amperometry & $<1 \mu \mathrm{g} / \mathrm{ml}$ & {$[11]$} \\
Colorimetric detection & $28.5 \mathrm{ng} / \mathrm{ml}$ & This work
\end{tabular}

${ }^{\text {a }}$ High performance liquid chromatography.

b Electrochemical detection.

c Surface plasma resonance.

d Molecularly imprinted polymer.

determine the relevant binding isotherms, batch rebinding experiments referred to as molecularly imprinted sorbent assays (MIAs) [15] and resembling typical immunoassays are usually employed. For instance, MIAs with radioactive stains (radio-ligand binding assays) have been utilized to determine the association constant and binding site density of the MIP of MO [13,14]. Despite the MIA being a well-established technique, there is a recent trend for detection modes other than radioactivity measurements being highly warranted [12]. Therefore, this work aimed at developing a colorimetric staining method to characterize MO-MIP binding.

An assay based on colorimetric staining offers advantages of costs and convenience, compared to those based on radioactive [13,14], fluorescent [16], enzymatic [17], and redox [18] reagents. In this work, the MO-bound MIP was brought into contact with an aqueous mixture of $\mathrm{Fe}^{3+}$ and $\left[\mathrm{Fe}(\mathrm{CN})_{6}\right]^{3-}$ so that the 3-phenolic group of MO was oxidized [19] and $\mathrm{Fe}^{3+}$ was reduced to $\mathrm{Fe}^{2+}$. As a result, the MO-bound MIP was stained with Prussian blue (PB), which was attributed to the instant co-precipitation of $\mathrm{Fe}^{2+}$ and $\left[\mathrm{Fe}(\mathrm{CN})_{6}\right]^{3-}\left(K_{\mathrm{sp}}=10^{-40}\right)$ [20]. Note: Either soluble PB $\left(\mathrm{KFe}\left[\mathrm{Fe}(\mathrm{CN})_{6}\right]\right)$ or insoluble PB $\left(\mathrm{Fe}_{4}\left[\mathrm{Fe}(\mathrm{CN})_{6}\right]_{3}\right)$ was formed. The formation of $\mathrm{PB}$ depends on whether or not $\mathrm{K}^{+}$participates in the co-precipitation. The staining mechanism is illustrated schematically in Fig. 1. Accordingly, the MO-bound MIP (which was dyed blue) could be easily distinguished, even by the naked eye, from the MIP without MO (yellowish). This also allowed us to quantify MO-MIP binding by visible spectroscopy. In addition, we also observed that such staining could distinguish MO from codeine. In this paper, the above-mentioned colorimetric detection of MO is discussed. In addition, physical properties of MIP and non-MIP particles, including their particle size distributions, binding site densities, and adsorption isotherms, are also compared.

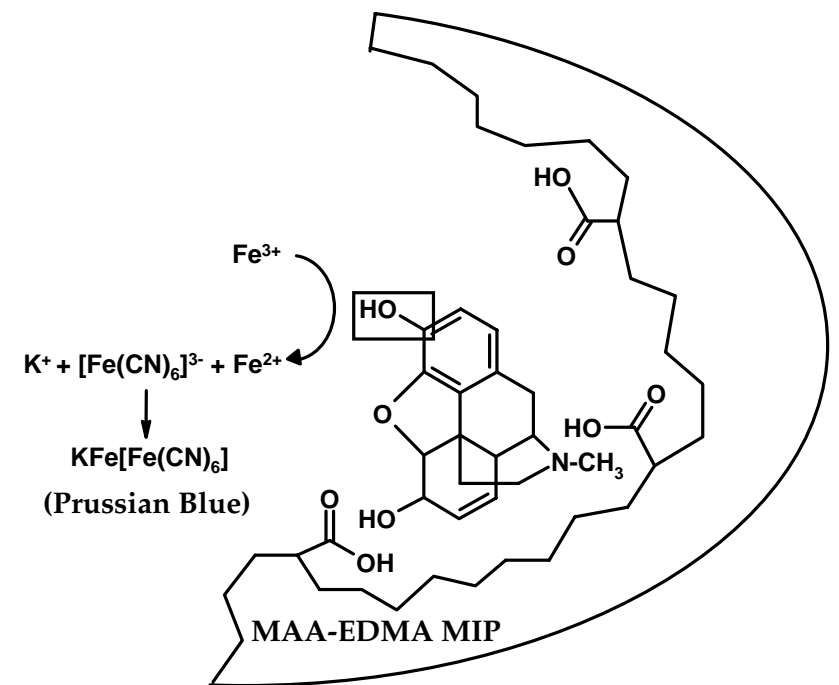

Fig. 1. Schematic illustration for the colorimetric staining in a MO-bound MIP by an aqueous mixture of $\mathrm{K}^{+}, \mathrm{Fe}^{3+}$, and $\left[\mathrm{Fe}(\mathrm{CN})_{6}\right]^{3-}$. MO acts as a reducing agent which bring about the precipitation of $\mathrm{PB}$ within the polymer matrix, while $\mathrm{PB}$ acts as a colorimetric reporter. The precipitation of $\mathrm{KFe}\left[\mathrm{Fe}(\mathrm{CN})_{6}\right]$ is known as soluble $\mathrm{PB}$. In the absence of $\mathrm{K}^{+}$, the formation of insoluble $\mathrm{PB}\left(\mathrm{Fe}_{4}\left[\mathrm{Fe}(\mathrm{CN})_{6}\right]_{3}\right)$ can also take place.

\section{Experimental}

\subsection{Reagents and standards}

All of the chemicals used in this work were either ACS reagent grade or analytical reagent grade and were used as received without further purification. Morphine in the form of MO hydrochloride and codeine in the form of tablet codeine phosphate $(15 \mathrm{mg})$ were both supplied by the National Bureau of Controlled Drugs, Department of Health, Taiwan (Taipei, Taiwan). Ethylene glycol dimethacrylate (EDMA, 98\%), $\mathrm{K}_{3} \mathrm{Fe}(\mathrm{CN})_{6}(>99 \%)$, and $\mathrm{FeCl}_{3} \cdot 6 \mathrm{H}_{2} \mathrm{O}(97 \%)$ were all purchased from Aldrich (Milwaukee, WI, USA). Acetonitrile (MeCN, 100\%) and acetic acid $(\mathrm{AcOH}, 100 \%)$ were both purchased from J.T. Baker (Phillipsburg, NJ, USA). Methanol (MeOH, 99.8\%), $\mathrm{HCl}(32 \%)$, methacrylic acid (MAA, 99\%), and 2.2'-azobis-isobutyronitrile (AIBN, 99\%) were purchased from Riedel-de Haën (Seelze, Germany), Merck (Darmstadt, Germany), Lancaseter (Morecambe, UK), and Showa (Tokyo, Japan), respectively. Deionized water (DIW) was used throughout this work. Unless especially noted, experiments were done at room temperature and in air.

\subsection{Spectroscopic detections of MO and codeine solutions with and without staining}

The UV-Vis spectra, which ranged from 200 to $900 \mathrm{~nm}$, of the following solutions were collected with a spectrophotometer (Model V-570, Jasco, Tokyo, Japan): (A) aqueous MO solutions stained by PB; (B) unreacted, diluted (51 times) staining solution; (C) aqueous MO solutions without 
staining; (D) $\mathrm{MO}$ in $\mathrm{MeOH} / \mathrm{AcOH} /$ water (volume ratio = $4: 1: 1$ ); (E) aqueous codeine solutions stained by PB; and (F) aqueous codeine solutions without staining. In particular, spectra A, C, and D were measured with various $\mathrm{MO}$ concentrations in order to obtain the calibration curves (plots of absorbances versus MO concentrations). The undiluted staining solution was composed of $20 \mathrm{mM} \mathrm{FeCl}_{3 \text { (aq) }}, 20 \mathrm{mM}$ $\mathrm{K}_{3} \mathrm{Fe}(\mathrm{CN})_{6(\mathrm{aq})}$, and a few drops of $\mathrm{HCl}$ which stabilized the mixture and resulted in a $\mathrm{pH}$ value of 1.57 . When staining, $0.2 \mathrm{ml}$ of the above-described aqueous mixture was dropped into $10 \mathrm{ml}$ of an $\mathrm{MO}$ or codeine solution of known concentration. Afterwards, the MO solution was dyed blue, and spectroscopic detection was carried out.

\subsection{Preparation of MIP and non-MIP particles}

The MO-MIP was prepared through the thermal radical copolymerization of MAA and EDMA in the presence of MO templates. The MO-MIP was used to selectively bind MO and then after separation of the MIP and the sample solution the staining procedure would take place. Our procedures were modified from those reported in the literature $[13,14]$ and are described below. Prior to polymerization, $2 \mathrm{mmol} \mathrm{MO}$ and $30 \mathrm{mmol}$ MAA were both dissolved in $30 \mathrm{ml} \mathrm{MeCN}$ and were stirred ultrasonically for $5 \mathrm{~min}$. Then, $120 \mathrm{mmol}$ EDMA and $280 \mathrm{mg}$ of AIBN were added. After 10 min of bubbling with $\mathrm{N}_{2}$, the beaker containing the monomer solution was covered and cured at $60{ }^{\circ} \mathrm{C}$ for $8 \mathrm{~h}$ in a water bath controlled by a thermostat (Model T-80, Tokyo Rikskikai, Tokyo, Japan). Finally, the dried polymeric monolith was thoroughly pulverized into fine particles with a pulverizer (Hi-Lyte, Taipei, Taiwan) or was exhaustively ground in a mortar, following vacuum drying at $40{ }^{\circ} \mathrm{C}$ for $8 \mathrm{~h}$. In addition, the non-MIP particles were also prepared according to the above procedures except for the absence of MO templates during polymerization. The as-prepared MIP particles were washed with an excess amount of the $4: 1: 1$ (v/v/v) $\mathrm{MeOH} / \mathrm{AcOH} /$ water (referred to as solution D in Section 2.2) several times until MO template was removed as much as possible, then the particles were washed with methanol and finally dried in a vacuum $[13,14]$. The removal of MO was assured by UV-Vis spectroscopy. The manually ground particles (referred to as "rough" particles), which quickly settled out in water, were characterized by a flow system (see Section 2.4). The mechanically pulverized particles (referred to as "fine" particles), which packed compactly but suspended well in $\mathrm{H}_{2} \mathrm{O}$, were used in the MIAs (see Section 2.5). The particle size distributions of both particles in DIW were analyzed with a laser particle size analyzer (Model LS230, Beckman Coulter, Fullerton, CA, USA).

\subsection{Flow-system characterizations of rough MIP and non-MIP particles}

To determine the binding site densities of rough MIP and non-MIP particles, a flow-system characterization, ana- $\log$ to chromatography, was employed. At the beginning, the MO templates resides in the as-ground MIP particles were washed until the level was dropped below the detection limit of the analytical method. Then the post-washed MIP particles re-adsorbed MO in $1.17 \mathrm{mM} \mathrm{MO}_{(\mathrm{aq})}$ until saturation. The same adsorption procedure was performed on the non-MIP particles. Finally, either $0.5 \mathrm{~g}$ MO-adsorbed MIP particles or $0.5 \mathrm{~g}$ MO-adsorbed non-MIP particles were packed in a polypropylene (PP) empty column with an inner diameter of $1.25 \mathrm{~cm}$ and a length of $5 \mathrm{~cm}$. Both ends of the column were equipped with filter papers and were connected to the PP tubing of a micro-pump system. Simultaneous monitoring of the outlet MO concentration was done by a UV-Vis spectrophotometer while the washing reagent (4:1:1 $\mathrm{MeOH} / \mathrm{AcOH} /$ water) was flowed through the packed column.

\subsection{Molecularly imprinted sorbent assays of fine MIP and non-MIP particles}

Molecularly imprinted sorbent assays with colorimetric detection (MO-particle binding stained by $\mathrm{PB}$ ) were applied to determine the adsorption isotherms of fine MIP and non-MIP particles. The MIA at each MO concentration was implemented in two separate steps: incubation and colorimetric detection. Incubation was carried out by mixing $5 \mathrm{mg}$ of post-washed MIP or non-MIP particles into $10 \mathrm{ml}$ of $\mathrm{a} \mathrm{MO}_{(\mathrm{aq})}$ solution with a definite concentration for $15 \mathrm{~h}$. The concentration of $\mathrm{MO}_{(\mathrm{aq})}$ varied from 50 to $50 \mathrm{mM}$. After incubation, the particles were filtered out and dried in a vacuum chamber. Colorimetric detection measured the absorbances at $746 \mathrm{~nm}$ (the characteristic wavelength of the PB) for the aqueous suspensions of stained, post-incubated particles. Colorimetric detection was carried out by adding $0.2 \mathrm{ml}$ of the staining solution into a $10 \mathrm{ml}$ aqueous suspension which contained $0.1 \mathrm{~g} / \mathrm{ml}$ post-incubated particles.

\section{Results and discussion}

\subsection{Spectra of MO and codeine solutions in the absence and presence of $\mathrm{Fe}^{3+}$ and $\left[\mathrm{Fe}(\mathrm{CN})_{6}\right]^{3-}$}

As illustrated in Fig. 1, the phenolic group at the 3-position of $\mathrm{MO}$ acts as a reducing agent and reduce $\mathrm{Fe}^{3+}$ to $\mathrm{Fe}^{2+}$, which then immediately co-precipitates with $\left[\mathrm{Fe}(\mathrm{CN})_{6}\right]^{3-}$ to yield $\mathrm{PB}$ within the polymer matrix. Therefore, $\mathrm{PB}$ serves as a reporter and achieves the colorimetric detection of MO. It is noted here that the orientation of the phenolic $\mathrm{OH}$ depicted in Fig. 1 is only to indicate the "free" $\mathrm{OH}$ available to the staining reagent. In general, some phenolic $\mathrm{OH}$ would be hydrogen-bonded to the polymer, and the reagent would have no access to the phenolic $\mathrm{OH}$. Similar situation has been observed for hydroxyl group in a polymer-bound sterol [21] and no hydroxyl groups would be free to react. This is due to the formation of covalent bond at 


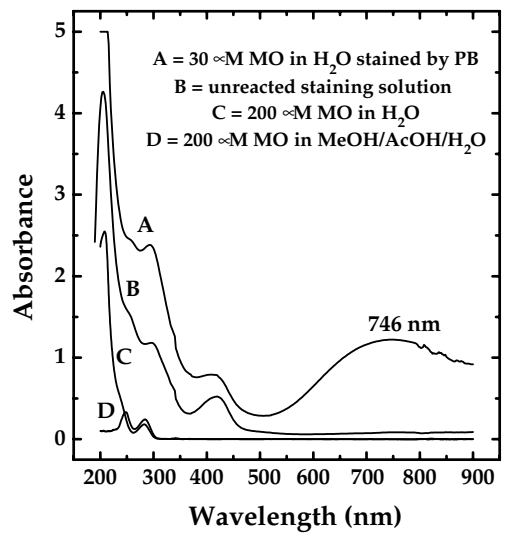

Fig. 2. The UV-Vis spectra of MO solutions with and without colorimetric staining: (A) $10 \mathrm{ml}$ of $30 \mu \mathrm{M} \mathrm{MO}_{(\mathrm{aq})}$ stained by $0.2 \mathrm{ml}$ of $20 \mathrm{mM}$ $\mathrm{FeCl}_{3(\mathrm{aq})}$ and $\mathrm{K}_{3} \mathrm{Fe}(\mathrm{CN})_{6(\mathrm{aq})}\left(\mathrm{pH} \mathrm{1.57)}\right.$; (B) $0.2 \mathrm{ml}$ of $20 \mathrm{mM} \mathrm{FeCl}_{3(\mathrm{aq})}$

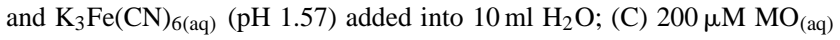
without staining; (D) $200 \mu \mathrm{M} \mathrm{MO}$ in $4: 1: 1$ (v/v/v) $\mathrm{MeOH} / \mathrm{AcOH} /$ water without staining.

the recognition site. According to the literature [19], the phenolic $\mathrm{OH}$ group will be oxidized to form pseudomorphine. Fig. 2 compares the UV-Vis spectra of MO solutions with and without colorimetric staining. It can be seen that the MO aqueous solution stained by an aqueous mixture of $\mathrm{FeCl}_{3}$ and $\mathrm{K}_{3} \mathrm{Fe}(\mathrm{CN})_{6}$ shows an adsorption band at ca. $746 \mathrm{~nm}$ (spectrum A). However, neither the MO aqueous solution (spectrum C) nor $\mathrm{MO}$ in 4:1:1 $\mathrm{MeOH} / \mathrm{AcOH} /$ water (spectrum D) exhibited any adsorption in the visible region despite both of them having intense absorption in the UV region. Presumably, the absorption at ca. $746 \mathrm{~nm}$ in spectrum A is in response to the formation of $\mathrm{PB}$, although a PB film typically features an absorption band at ca. $690 \mathrm{~nm}$ [22]. As a consequence, the colorimetric staining of $\mathrm{MO}$ by $\mathrm{PB}$, which allows visualization of the MO concentration, is demonstrated. In addition, by comparing the peak heights and concentrations of spectra A, C, and D in Fig. 2, it can be found that the colorimetric staining enhances not only the sensitivity but also the spectroscopic detection limit of MO. On the basis of the calibration curves, Fig. 3 illustrates the above findings.

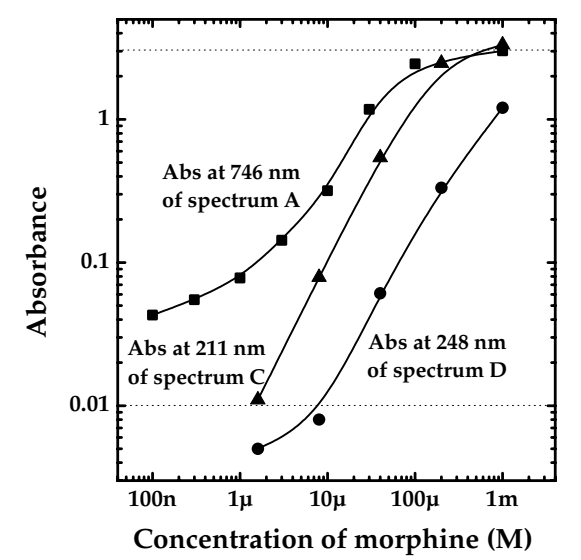

Fig. 3. Calibration curves for the spectroscopic detections of MO in Fig. 2.

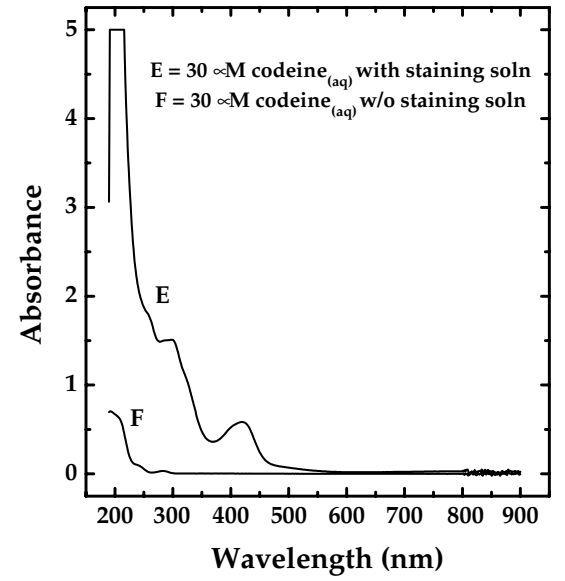

Fig. 4. The UV-Vis spectra of codeine solutions in the presence and absence of staining solution. (E): $10 \mathrm{ml}$ of $30 \mu \mathrm{M}$ codeine $(\mathrm{aq})$ added with $0.2 \mathrm{ml}$ of $20 \mathrm{mM} \mathrm{FeCl}_{3(\mathrm{aq})}$ and $\mathrm{K}_{3} \mathrm{Fe}(\mathrm{CN})_{6(\mathrm{aq})}(\mathrm{pH} 1.57)$; (F): $30 \mu \mathrm{M}$ codeine $_{(\mathrm{aq})}$ without (w/o) the staining solution.

It is shown in Fig. 3 that the MO solution after staining (curve A) displays higher absorbances than those of unstained MO solutions (curves C and D). Moreover, since the absorbance readings are considered reliable when they range between 0.01 and 3.00, the detection limits for each case are thus estimated as follows: case A: $100 \mathrm{nM}$ to $1 \mathrm{mM}$; case C: $1 \mu \mathrm{M}$ to $1 \mathrm{mM}$; and case D: $10 \mu \mathrm{M}$ to $3 \mathrm{mM}$ (Note: $1 \mathrm{mM}=0.285 \mathrm{mg} / \mathrm{ml}$ for $\mathrm{MO}$ ). This indicates that the colorimetric detection of MO by PB staining is more sensitive than does the ordinary UV detection. It was also determined that this colorimetric staining possesses a promising detection limit for MO $(28.5 \mathrm{ng} / \mathrm{ml})$, as compared to values from the literature summarized in Table 1. In addition, it was discovered that the staining could differentiate between MO and codeine. Codeine has a very similar molecular structure to MO except for replacing 3-phenol by a 3-methyloxide group $[13,14]$. Fig. 4 shows the spectra of codeine aqueous solutions with and without staining. It can be seen that neither spectra display an absorption in the visible region. This is because codeine lacks the oxidizable phenol group at the 3-position and cannot reduce $\mathrm{Fe}^{3+}$ to $\mathrm{Fe}^{2+}$. As a consequence, it is presumed that colorimetric staining can discriminate other MO analogs which lack the 3-phenol group, such as heroin and pholcodine.

\subsection{Particle size distributions of MIP particles}

The two approaches we adopted to process the as-polymerized monoliths into particles were described in Section 2: manual grinding in a mortar and mechanical pulverization. Particle size distributions of the particles obtained from these two processes are compared in Fig. 5. Undoubtedly, the manually ground (rough) particles were much larger than the mechanically pulverized (fine) ones. The rough particles had a volume-averaged particle diameter of $252 \mu \mathrm{M}$, and $80 \%$ of them had a particle diameter ranging from 


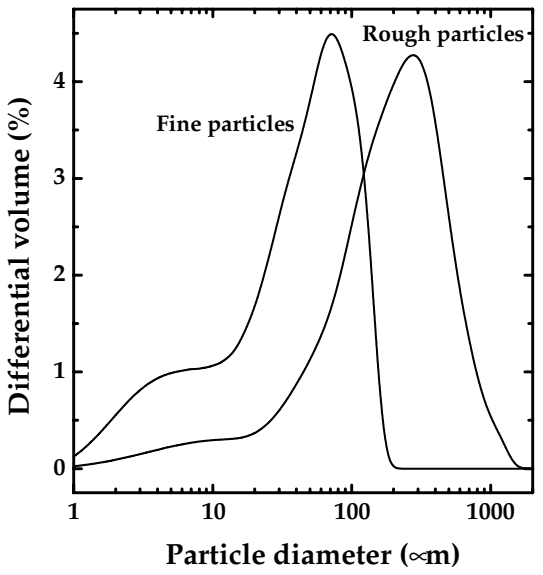

Fig. 5. Particle size distributions of mechanically pulverized (fine) and manually ground (rough) MIP particles.

$37.2 \mu \mathrm{M}(10 \%)$ to $530 \mu \mathrm{M}(90 \%)$. The fine particles showed a volume-averaged particle diameter of $51.5 \mu \mathrm{M}$, and $80 \%$ of them had a particle diameter ranging from $4.68 \mu \mathrm{M}(10 \%)$ to $110 \mu \mathrm{M}(90 \%)$. (Note: The volume-averaged value is generally larger than the number-averaged value.) Presumably, there was no significant difference between the particle size distributions of MIP and non-MIP particles. Using different approaches without sieving, we attempted to preliminarily compare the effects of particle size on MO-MIP binding.

\subsection{Binding site densities of rough MIP and non-MIP particles}

The flow-system characterization described in Section 2.4 was applied to determine the binding site densities of rough MIP and non-MIP particles. By using calibration curve D in Fig. 3, the amounts of MO washed out from a packed bed of MO-adsorbed MIP or non-MIP were simultaneously monitored. The results of the flow-system characterizations of MIP and non-MIP particles are respectively plotted in Fig. 6. It can be determined from this plot that binding site densities of rough MIP and non-MIP particles are ca. 55 and $20 \mu \mathrm{mol} / \mathrm{g}$, respectively. That is, rough MIP particles possess a binding site density 2.75 times that of rough non-MIP particles. This verifies that molecular imprinting was successful. Yet, the binding site densities determined here could be underestimated, since both MIP and non-MIP particles adsorbed $\mathrm{MO}$ in solution of $1.17 \mathrm{mM} \mathrm{MO}(\mathrm{aq})$ which is not very concentrated. To precisely determine binding site densities, adsorption isotherms were needed.

\subsection{Adsorption isotherms of fine MIP and non-MIP particles obtained from MIAs}

Fig. 7 gives the results of MIAs on fine, post-washed MIP and non-MIP particles. As can be seen in this figure, the MO-adsorbed MIP exhibits higher absorbances at ca. $746 \mathrm{~nm}$ than does the MO-adsorbed non-MIP. Furthermore,

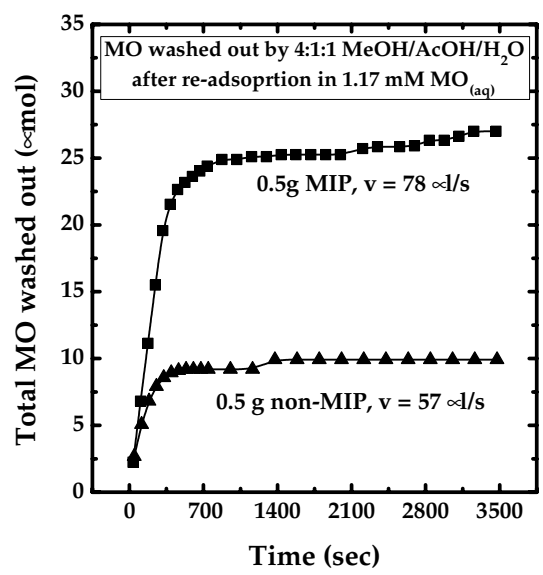

Fig. 6. Continuous wash of the re-adsorbed MIP and non-MIP particles with 4:1:1 MeOH/AcOH/water. The molar numbers of MO washed out were determined by monitoring the change in the absorbance at $248 \mathrm{~nm}$ and with the help of calibration curve D given in Fig. 3.

MIP behaves differently from non-MIP. The absorbance of non-MIP increased exponentially with MO concentration, whereas the absorbance curve of MIP exhibited a reflecting point at ca. $500 \mu \mathrm{M}$. Presumably, non-MIP follows one-site binding, while MIP obeys two-site binding [13]. To obtain realistic adsorption isotherms, Fig. 7 was converted to Fig. 8 with the aid of calibration curve A in Fig. 3. Since the spectroscopic data obtained at $50 \mathrm{mM}$ were less reliable (absorbance > 3), curve fitting was done for data which ranged from $50 \mathrm{nM}$ to $5 \mathrm{mM}$. As judged from Fig. 7, the oneand two-site binding models were used to fit the isotherms of non-MIP and MIP in Fig. 8, respectively. These two models are formulated as follows:

$b=\frac{B C}{K+C} \quad$ (one-site binding model),

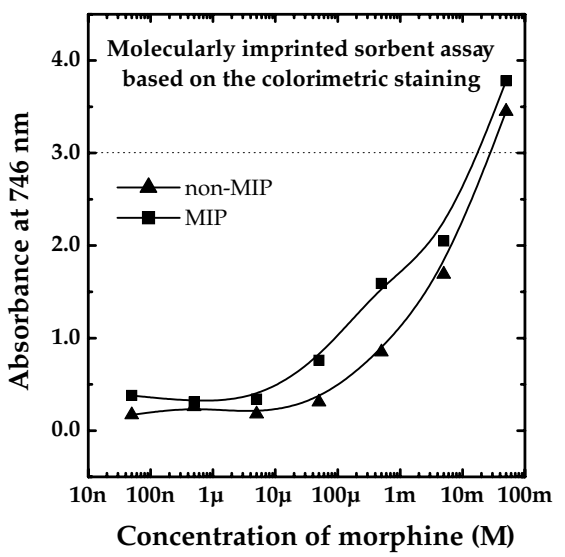

Fig. 7. Molecularly imprinted sorbent assay based on the colorimetric detection. Incubation conditions: adding $5 \mathrm{mg}$ washed polymer particles into $10 \mathrm{ml} \mathrm{MO}$ aqueous solution and then incubated for $15 \mathrm{~h}$; staining conditions: adding $0.2 \mathrm{ml}$ of $20 \mathrm{mM} \mathrm{FeCl}_{3(\mathrm{aq})}$ and $\mathrm{K}_{3} \mathrm{Fe}(\mathrm{CN})_{6(\mathrm{aq})}(\mathrm{pH}$ 1.57 ) into $10 \mathrm{ml}$ of $0.1 \mathrm{mg} / \mathrm{ml}$ post-incubated, aqueous polymer suspension. 


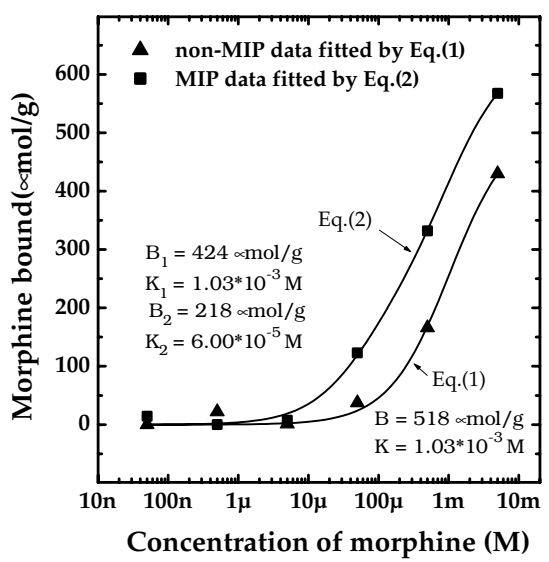

Fig. 8. Quantitative analysis of the MIA results obtained in Fig. 7. The molar numbers of MO washed out were determined by comparing Fig. 7 with the help of calibration curve A given in Fig. 3. The effects of particle scattering were compensated prior to the use of calibration curve A.

and

$b=\frac{B_{1} C}{K_{1}+C}+\frac{B_{2} C}{K_{2}+C} \quad$ (two-site binding model),

where $b$ is the molar number of MO adsorbed per weight of particles ( $\mu \mathrm{mol} / \mathrm{g}), B$ denotes the binding site density for MO ( $\mu \mathrm{mol} / \mathrm{g}), K$ represents the dissociation constant $\left(\mathrm{M}^{-1}\right)$, and $C$ stands for the molar concentration (M) of MO in the incubation medium. In Eq. (2), subscripts 1 and 2 are used to denote properties for the two classes of binding sites. Basically, Eq. (1) represents the typical Langmuir adsorption isotherm, where adsorption is assumed to contain only one type of site. Eq. (2) is employed to present a modified Langmuir adsorption isotherm in which two classes of binding sites exist.

The well-fitted results in Fig. 8 indicate that fine non-MIP particles possess a binding site density $(B)$ of $518 \mu \mathrm{mol} / \mathrm{g}$ and a dissociation constant $(K)$ of $1.03 \times 10^{-3} \mathrm{M}$, whereas fine MIP particles contain two classes of sites with the following physical properties: $B_{1}$ of $424 \mu \mathrm{mol} / \mathrm{g}$ and $K_{1}$ of $1.03 \times 10^{-3} \mathrm{M}$, and $B_{2}$ of $218 \mu \mathrm{mol} / \mathrm{g}$ and $K_{2}$ of $6.00 \times 10^{-5} \mathrm{M}$. As those values were obtained from fitting the experimental data with either one- or two-site binding model, they should be more realistic when comparing to the theoretical values calculated by assuming a complete polymerization. Accordingly, fine MIP particles have a total binding site density of $642 \mu \mathrm{mol} / \mathrm{g}\left(B_{1}+B_{2}\right)$. The MIP's two-site binding reflects that the MO was adsorbed on either imprinting sites or non-imprinting sites. Non-imprinting binding is simply attributed to the non-covalent interaction between the carboxylic group of poly-MAA and the phenolic group of MO, by which MO binding on non-MIP particles takes place. Such non-imprinting binding exhibits a lower binding affinity with a dissociation constant of $1.03 \times 10^{-3} \mathrm{M}$, as compared to imprinting binding $\left(K_{2}\right.$ of $\left.6.00 \times 10^{-5} \mathrm{M}\right)$. Despite the lower affinity, non-imprinting sites still make up the majority of sites on MIP. Similar results can be found in the literature [13].
In addition, by comparing Fig. 8 (results of fine particles with an average diameter of $51.5 \mu \mathrm{m}$ ) with Fig. 6 (results of rough particles with an average diameter of $252 \mu \mathrm{m}$ ), the particle size effects on MO-MIP binding can be preliminarily deduced. Fig. 8 reveals that the binding amounts on fine MIP and non-MIP particles were 433 and $275 \mu \mathrm{mol} / \mathrm{g}$, respectively, when the incubated concentration was $1.17 \mathrm{mM}$. Obviously, the above values are much larger than those obtained in Fig. 6. But the binding ratio of MIP to non-MIP obtained here was 1.57 , which is smaller than the case with rough particles (2.75). Accordingly, fine particles are considered to contribute a higher binding site density due to their larger surface area, but the reduction in particle size leads to a decrease in the ratio of imprinting to non-imprinting sites.

\section{Conclusions}

This work reports colorimetric detection of MO in an MIP. The successful staining of MO-bound MIP was achieved with the help of an aqueous mixture containing $\mathrm{Fe}^{3+}$ and $\left[\mathrm{Fe}(\mathrm{CN})_{6}\right]^{3-}$. The main conclusions from this study are summarized here.

1. Morphine can be stained by $\mathrm{PB}$ with an aqueous mixture of $\mathrm{Fe}^{3+}$ and $\left[\mathrm{Fe}(\mathrm{CN})_{6}\right]^{3-}$. Such colorimetric staining allows spectroscopic detection of MO by measuring the absorbance change at $746 \mathrm{~nm}$, which is visible even to the naked eye. In addition to the advantages of costs and convenience, the staining also provides promising sensitivity with a detection limit of $100 \mathrm{nM}$ and can discriminate MO analogs which lack the oxidizable 3-position phenol, such as codeine.

2. MIP-MO binding obeys the two-site-binding mechanism, whereas non-MIP-MO binding follows the simple Langmuir adsorption isotherm. MIAs based on colorimetric staining reveal that the binding site density and dissociation constant for non-MIP are $518 \mu \mathrm{mol} / \mathrm{g}$ and $1.03 \times$ $10^{-3} \mathrm{M}$, respectively. In comparison, MIP features two classes of sites with the following physical properties: $B_{1}$ of $424 \mu \mathrm{mol} / \mathrm{g}$ and $K_{1}$ of $1.03 \times 10^{-3} \mathrm{M}$, and $B_{2}$ of $218 \mu \mathrm{mol} / \mathrm{g}$ and $K_{2}$ of $6.00 \times 10^{-5} \mathrm{M}$. ( $B$ and $K$ represent the binding site density and dissociation constant, respectively. Subscripts 1 and 2 denote non-imprinting and imprinting sites of MIP, respectively.)

3. Fine MIP particles can contribute a higher binding site density due to their larger surface area, but reducing the particle size of MIP results in a decrease in the ratio of imprinting to non-imprinting sites.

\section{Acknowledgements}

Helpful discussions with Professor Tse-Chuan Chou of the Department of Chemical Engineering, Professor Shu-Chu Shieh of the Department of Medical Technology, 
and Xi-Zhang Lin, of the Department of Internal Medicine, all of National Cheng Kung University, Tainan, Taiwan, are greatly appreciated. Experimental assistance from $\mathrm{Mr}$. Chung-Wei Yang is also deeply appreciated. This research was supported by the Program for Promoting Academic Excellence of Universities, sponsored by the Ministry of Education of the Republic of China, under grant no. EX-91-E-FA09-5-4.

\section{References}

[1] C.F. Mclean, L.E. Mather, P.A. Sloan, J. Pharm. Pharmacol. 42 (1990) 669.

[2] J.O. Svensson, A. Rane, J. Sawe, F. Sjoquist, J. Chromatogr. 230 (1982) 427.

[3] M. Arunyanart, L.J. Cline Love, J. Chromatogr. 342 (1985) 293.

[4] R.D. Abbott, A. Townshend, R. Gill, Analyst 112 (1987) 397.

[5] J. Gerostamoulos, K. Crump, I.M. Mclntyre, O.H. Drummer, J. Chromatogr. 617 (1993) 152.

[6] P.P. Rop, F. Grimaldi, J. Burle, M.N. De Saint Leger, A. Viala, J. Chromatogr. B 661 (1994) 245.

[7] W.-J. Liaw, S.-T. Ho, J.-J. Wang, O.Y.-P. Hu, J.-H. Li, J. Chromatogr. B 714 (1998) 237.

[8] J. Beike, G. Blaschke, A. Mertz, H. Kohler, B. Brinkmann, Int. J. Legal Med. 112 (1998) 8.
[9] G. Sakai, K. Ogata, T. Uda, N. Miura, N. Yamazoe, Sens. Actuators B 49 (1998) 5.

[10] D. Kriz, K. Mosbach, Anal. Chim. Acta 300 (1995) 71.

[11] K.A. Johnson, D. Kriz, Instrum. Sci. Technol. 25 (1997) 29.

[12] B. Sellergren (Ed.), Molecularly Imprinted Polymers: Man-made Mimics of Antibodies and Their Applications in Analytical Chemistry-Techniques and Instrumentation in Analytical Chemistry, vol. 23, Elsevier, Amsterdam 2001.

[13] L.I. Andersson, R. Müller, G. Vlatakis, K. Mosbach, Proc. Natl. Acad. Sci. U.S.A. 97 (1995) 4788.

[14] A.G. Mayes, C.R. Lowe, in: E. Reid, H.M. Hill, I.D. Wilson (Eds.), Methodological Surveys in Bioanalysis of Drugs, vol. 25, Drug Development Assay Approaches Including Molecular Imprinting and Biomarkers, The Royal Society of Chemistry, Cambridge, UK, 1998, p. 28.

[15] G. Vlatakis, L.I. Andersson, R. Müller, K. Mosbach, Nature 361 (1993) 645.

[16] K. Haupt, A.G. Mayes, K. Mosbach, Anal. Chem. 70 (1998) 3936.

[17] I. Surugiu, L. Ye, E. Yilmaz, A. Dzgoev, B. Danielsson, K. Mosbach, K. Haupt, Analyst 125 (2000) 13.

[18] S. Kröger, A.P.F. Turner, K. Mosbach, K. Haupt, Anal. Chem. 71 (1999) 3698.

[19] P.H. Jordan, J.P. Hart, Analyst 116 (1991) 991.

[20] A. G. Sharpe, The Chemistry of Cyano Complexes of the Transition Metals, Academic Press, London, UK, 1976 (Chapter 7).

[21] C. Alexander, C.R. Smith, M.J. Whitecombe, E.N. Vulfson, J. Am. Chem. Soc. 121 (1999) 6640.

[22] K. Itaya, I. Uchida, V.D. Neff, Acc. Chem. Res. 19 (1986) 162. 